\title{
Effects of Radiotherapy on the Risk of Developing Secondary Malignant Neoplasms in Hodgkin's Lymphoma Survivors
}

\author{
Tamara Abou-Antoun ${ }^{1}$, Rachelle Mikhael ${ }^{1 \&}$, Marcel Massoud ${ }^{2 \&}$, Georges \\ Chahine $^{2}$, Aline Saad ${ }^{3 *}$
}

\begin{abstract}
Extended follow-up of Hodgkin lymphoma (HL) survivors indicates that these patients are at high risk of secondary malignant neoplasms (SMNs) contributing to increased morbidity and mortality. This study examined the characteristics of HL survivors who developed SMNs with the aim to report any correlation with radiotherapy (RT) dose. In this retrospective multi-center cohort study of HL patients treated between 1990 and 2011 at three major teaching hospitals in Lebanon, classification was into two groups including those treated with combined modality (RT and chemotherapy-CHT) and those treated with CHT alone. Approval from the University Institutional Review Board (IRB) was obtained. Of the 112 patients evaluated, 52.7\% (59) received the combined modality while $47.3 \%$ (53) received CHT alone. There were 6 cases of SMNs in the combined modality cohort and 5 cases in the CHT cohort. The mean RT dose in the combined modality cohort was 34.5 Gray (Gy) (SD \pm 5.3). A statistically significant increase (1.5 fold) in the risk of developing SMNs was observed among patients who received a dose higher than $41 \mathrm{~Gy}$ compared to a dose between 20 to $30 \mathrm{~Gy}$ (OR=1.5; $95 \%$ confidence interval= 0.674 to $3.339, p=0.012$ ). The risk of SMNs was not significantly higher among patients who received extended field compared to involved field $\mathrm{RT}(\mathrm{p}=\mathbf{0 . 9 6 4}$ ). This study showed that the risk of developing SMNs is higher among patients treated with RT dose greater than $31 \mathrm{~Gy}$, independent of the RT type used.
\end{abstract}

Keywords: Secondary malignant neoplasms - Hodgkin's lymphoma - radiotherapy - extended field radiation therapy

Asian Pac J Cancer Prev, 17 (2), 749-754

\section{Introduction}

During the past few decades, significant progress has been achieved in the treatment of Hodgkin's lymphoma (HL) patients. This disease has now become a curable malignancy in at least $80 \%$ of its patients (van Leeuwen et al., 2000; Noordijk et al., 2006; Matasar et al., 2008; NCCN, 2015). In fact, when compared to other cancers, the 5-year survival rates of HL patients remain unmatched for the past 4 decades (NCCN, 2015). HL patients have an overwhelming chance of cure due to the improvement of treatment strategies and the use of combined modality therapy including radiotherapy (RT) and chemotherapy (CHT) (van Leeuwen et al., 2000; Noordijk et al., 2006; Matasar et al., 2008; NCCN, 2015).

However, this success in cure rates has been dimmed by the treatment associated long-term toxicity (Omer et al., 2012). Accordingly, the care of HL patients extends for years beyond treatment to encompass these long-term toxicities which are contributing to overall morbidity and mortality (Constine et al., 2008; Yahalom, 2009). The most serious long-term toxicities documented in survivors of HL include secondary malignant neoplasms (SMNs), cardiovascular diseases, hypothyroidism, and fertility issues (Lorigan et al., 2005; NCCN, 2015). Among SMNs, solid tumors were more commonly seen compared to hematologic malignancies (Aleman et al., 2012; NCCN, 2015). In fact, RT used as a component of first-line therapy in HL increased the risk of developing secondary solid tumors particularly affecting the lungs, breast, connective tissues and gastrointestinal tract (Constine et al., 2008; Oeffinger et al., 2013; Choi et al., 2014; NCCN, 2015). Secondary hematologic malignancies, including acute myelogenous leukemia/ myelodysplastic syndrome (AML/MDS) and Non-Hodgkin's Lymphoma (NHL), were mostly documented with CHT administration (Oeffinger et al., 2013; Choi et al., 2014; NCCN, 2015). Most studies showed that the appearances of secondary solid tumors were observed 10 to 15 years after RT exposure, while the peak incidence of secondary leukemia and NHL was 5 to 10 years after CHT (Schellong et al., 1997; Aleman et al., 2003; van Leeuwen et al., 2003;

${ }^{1}$ Department of Pharmaceutical Sciences, ${ }^{3}$ Department of Pharmacy Practice, School of Pharmacy, Lebanese American University, Byblos, ${ }^{2}$ Faculty of Medical Sciences, Holy Spirit University of Kaslik, USEK, Jounieh, Lebanon \&Equal contributors *For correspondence: aline.saad@lau.edu.lb 
Schellong and Riepenhausen, 2004).

Numerous studies have explored the factors affecting SMNs development in HL survivors with a focus on the evolving treatment plans, age at diagnosis and time since diagnosis (Ng et al., 2002; Franklin et al., 2006; Hodgson et al., 2007; O'Brien et al., 2010). Among those, the radiation dose has been identified as a relevant risk factor with this risk of SMNs being directly proportional to the radiation dose (Ng et al., 2002; Travis et al., 2002; Omer et al., 2012). Some studies showed an increased risk of breast and lung cancer at radiation dose $\geq 20$ Gray (Gy) (Gilbert et al., 2003, Travis et al., 2003; van Leeuwen et al., 2003). Travis et al. showed an 8-fold increased risk of breast cancer with a dose of more than 40 Gy (Travis et al., 2003). Yet, other publications raised doubt about the theory of lower-dose RT reducing the incidence of SMNs (O'Brien et al., 2010; Omer et al., 2012). As this correlation is still controversial and the corresponding data has been mostly reported in developed countries, the association between RT dose and SMNs is less known for developing countries, particularly in the Middle East.

Thus, the aim of this retrospective cohort study is to quantify the overall incidence of SMNs in HL survivors undergoing RT and CHT. Particularly, we wanted to examine the characteristics of HL survivors who developed SMNs and assess if a specific dose response correlation exists between RT doses and cancer induction. Secondary outcomes included an evaluation of the type of RT (Extended Field Radiation Therapy-EFRT versus Involved Field Radiation Therapy- IFRT) on the risk of SMNs development.

\section{Materials and Methods}

\section{Setting}

This retrospective multicenter cohort study consisted of one hundred and twelve patients treated for HL between 1990 and 2011 at three major Lebanese teaching hospitals in the Beirut and Byblos districts. Approval from the University Institutional Review Board (IRB) was obtained for this study.

\section{Procedure}

Patients were classified into 2 groups: those who received combined modality of RT and CHT and those who received CHT alone. Patients with a histologically confirmed diagnosis of HL were eligible for inclusion. Included patients were staged according to the Ann Arbor staging system (stage I to IV). All 4 subtypes of classical HL were included as well as lymphocyte-predominant Hodgkin Lymphoma (LPHL). Patients who died due to refractory disease or due to SMNs were also included in the study. Patient data were collected inclusively from 1990 until the end of December 2011.

\section{Data collection sheet}

Patient data regarding birth date, gender, age at diagnosis of HL, date of diagnosis, HL characteristics, type and dose of radiation, chemotherapy regimens and total number of administered cycles, follow-up duration, date of diagnosis of SMNs, and type of secondary cancers were obtained from the medical records at the treating hospitals.

\section{Statistical analysis}

The Pearson's chi-squared test $(\chi 2)$ was used to evaluate both primary and secondary outcomes. The analysis was conducted using the Statistical Package for Social Sciences (SPSS) software (version 19, Chicago, USA). Odds ratio (OR) and the corresponding confidence interval (CI) were calculated. Statistical significant was set at a $\mathrm{p}$ value $<0.05$.

\section{Results}

Characteristics of the cohort study

In this retrospective multi-institutional cohort study,

Table 1. Baseline Characteristics of Hodgkin Lymphoma Survivors

\begin{tabular}{|c|c|}
\hline Characteristics & No. $(\%)$ \\
\hline \multicolumn{2}{|l|}{ Gender } \\
\hline Female & $57(50.9)$ \\
\hline Male & $55(49.1)$ \\
\hline \multicolumn{2}{|l|}{ Clinical Stage } \\
\hline I & $17(15.2)$ \\
\hline II & $43(38.4)$ \\
\hline III & $21(18.8)$ \\
\hline IV & $11(9.8)$ \\
\hline Unknown & $20(17.8)$ \\
\hline \multicolumn{2}{|l|}{ General Symptoms } \\
\hline A & $45(40.2)$ \\
\hline $\mathrm{B}$ & $45(40.2)$ \\
\hline Unknown & $22(19.6)$ \\
\hline \multicolumn{2}{|l|}{ Histological type of Hodgkin's Lymphoma } \\
\hline Nodular sclerosis & $63(56.3)$ \\
\hline Mixed cellularity & $21(18.8)$ \\
\hline Lymphocyte-rich & $3(2.7)$ \\
\hline Lymphocyte-depleted & $1(0.9)$ \\
\hline Lymphocyte predominant & $2(1.8)$ \\
\hline Unknown & $22(19.5)$ \\
\hline \multicolumn{2}{|l|}{ Treatment category } \\
\hline Chemotherapy (CHT) only & $53(47.3)$ \\
\hline Combined Modality (Chemotherapy plus radiotherapy) & $59(52.7)$ \\
\hline \multicolumn{2}{|c|}{ Chemotherapy-CHT } \\
\hline ABVD & $64(77.1)$ \\
\hline MOPP & $2(2.41)$ \\
\hline BEACOPP & $1(1.2)$ \\
\hline $\mathrm{ABVD} / \mathrm{BEACOPP}$ & $1(1.2)$ \\
\hline ABVD/MOPP & $12(14.4)$ \\
\hline ABVD/NOVP & $1(1.2)$ \\
\hline ABVD/MOPP/NOVP & $1(1.2)$ \\
\hline ABVD/COPP/VIP & $1(1.2)$ \\
\hline \multicolumn{2}{|l|}{ Number of Chemotherapy Cycles } \\
\hline $1-3$ & $15(18.1)$ \\
\hline $4-6$ & $57(68.7)$ \\
\hline$>7$ & $11(13.3)$ \\
\hline \multicolumn{2}{|l|}{ Type of Radiotherapy-RT } \\
\hline Extended Field Radiation Therapy & $29(49.1)$ \\
\hline Involved Field Radiation Therapy & $28(47.4)$ \\
\hline Unknown & $2(3.39)$ \\
\hline
\end{tabular}

ABVD, adriamycin, bleomycin, vinblastine, dacarbazine; MOPP, chlorambucil, vinblastine, procarbazine, prednisone; BEACOPP, bleomycin, etoposide, adriamycin, cyclophosphamide, vincristine, procarbazine, prednisone; NOVP, mitoxantrone, vincristine, vinblastine, and prednisone; COPP, cyclophosphamide, vincristine, procarbazine, prednisone; VIP, etoposide or vinblastine, ifosfamide, cisplatin 
Effects of Radiotherapy on Risk of Developing Secondary Malignant Neoplasms in Hodgkin's Lymphoma Survivors

one hundred and twelve patients treated for HL between 1990 and 2011 were evaluated. Among them, fifty seven (50.9\%) were women and fifty five $(49.1 \%)$ were men. A detailed description of the characteristics of the study population is presented in Table 1 . The average age at diagnosis for HL was 31 years (range 7-79 years) with 95\% CI [28.41-34.92]. Among the 112 patients evaluated with HL, $18.75 \%$ (21 patients) were less than 21 years at diagnosis. The most common histological type was nodular sclerosis (56.3\%). According to the Ann Arbor's classification, the majority of patients had early stages (Stage I-II) representing $53.6 \%$ of the entire studied population. Advanced stages (Stage III-IV) and unknown stages frequencies were $28.6 \%$ and $17.9 \%$, respectively. The follow-up duration of these patients ranged from 1-27 years (median $=13$ years).

A total of 59 patients $(52.7 \%)$ received combined modality of RT and CHT while the other 53 patients (47.3\%) received CHT alone. Information related to chemotherapeutic regimens and number of cycles administered as well as the type of radiation field used (extended versus involved) were documented for the study population. Most patients $(77.11 \%)$ were treated with doxorubicin, bleomycin, vinblastine, and dacarbazine (ABVD) and had an average number of cycles between 4 and 6 cycles $(77.11 \%$ and $68.67 \%$ respectively). Among

Table 2. Secondary Malignant Neoplasms Development According to Treatment Modalities

\begin{tabular}{|c|c|c|}
\hline $\begin{array}{l}\text { Secondary Malignant } \\
\text { Neoplasms (SMNs) }\end{array}$ & $\begin{array}{l}\text { Chemotherapy } \\
\text { only } \\
\mathrm{N}=53 \mathrm{~N}(\%)\end{array}$ & $\begin{array}{l}\text { Chemotherapy } \\
\text { Plus Radiotherapy } \\
\text { ) } \mathrm{N}=59 \mathrm{~N}(\%)\end{array}$ \\
\hline \multicolumn{3}{|l|}{ Solid tumors } \\
\hline Breast & $1(1.89)$ & $4(6.78)$ \\
\hline Nasopharynx & - & $1(1.69)$ \\
\hline Thyroid & $1(1.89)$ & - \\
\hline Peritoneal Mesothelioma & - & $1(1.69)$ \\
\hline Small cell lung carcinoma & $1(1.89)$ & - \\
\hline Bladder diverticulum & $1(1.89)$ & - \\
\hline \multicolumn{3}{|l|}{ Hematologic tumors } \\
\hline Non Hodgkin T cell lymphoma & $1(1.89)$ & - \\
\hline
\end{tabular}

Table 3. Effect of Radiotherapy Dose on Secondary Malignant Neoplasms (SMNs)

\begin{tabular}{lcc}
\hline Dose of Radiation (Gray) & $\begin{array}{c}\text { No. of Patients }(\%) \\
(\mathrm{N}=59)\end{array}$ & $\begin{array}{c}\text { SMNs } \\
\text { No. }(\%)\end{array}$ \\
\hline Unknown & $2(3.39)$ & $0 \quad(0)$ \\
$20-30$ & $18(30.5)$ & $0 \quad(0)$ \\
$31-40$ & $36(61.1)$ & $5(13.9)$ \\
$41-50$ & $3(5.09)$ & $1(33.3)$ \\
$>51$ & $0(0)$ & 0 \\
\hline
\end{tabular}

those who received RT, approximately half of them received EFRT $(49.15 \%)$ while the other half received IFRT $(47.46 \%)$.

Primary outcomes: secondary cancers and RT-Dose response relationship

By December 2011, 11 SMNs had been diagnosed in 112 patients for a cumulative incidence, 13 years after the diagnosis of HL, of $9.8 \%$. Ten of these (90.9\% cases) were documented secondary solid tumors and one case $(9.1 \%)$ as NHL. Of the secondary solid tumors, 5 of the diagnoses $(45.5 \%)$ were breast cancers. The median age at diagnosis of SMNs was 45 years and ranged from 24-68 years. More women developed SMNs compared to men in the studied population ( 7 female vs 4 male). Two of the HL survivors who were less than 21 of age at diagnosis (9.5\%) developed SMNs.

For patients treated with combined modality, 6 out of fifty nine $(10 \%)$ developed secondary solid tumors with 4 cases of breast cancer, one nasopharynx and one peritoneal mesothelioma. The mean time from radiation to the development of secondary solid tumors was 14.1 years (Standard deviation $\mathrm{SD} \pm 8.35$ ) while the median time was 24 years (range 12-25). For patients treated with CHT alone, 5 out of fifty three $(9.4 \%)$ developed secondary cancers as detailed in Table 2 . The median time for the development of secondary cancers in the CHT cohort was 12 years (range 11-20). It was notable that secondary peritoneal mesothelioma was documented in the combined modality cohort and secondary thyroid cancer developed in a HL survivor in the CHT cohort.

The mean treatment dose among the fifty nine patients in cohort 1 who received RT was $34.5 \mathrm{~Gy}(\mathrm{SD} \pm 5.3)$. The risk of SMNs development was higher among those who received a radiation dose higher than $41 \mathrm{~Gy}$ than among those who received a total dose between 31 and 40 Gy (33.33\% versus $13.9 \%$ ). An increased risk of secondary cancers was also noted among those who received radiation dose between 31 to $40 \mathrm{~Gy}$ as compared to those who received a radiation dose between 20 to $30 \mathrm{~Gy}$; however the results did not reach statistically significance $(\mathrm{OR}=1.161 ; 95 \% \mathrm{CI}=1.019$ to $1.324, \mathrm{p}=0.097)$. Finally, a statistical significance increase (1.5 fold) in the risk of SMNs development was seen among patients who receive a dose higher than $41 \mathrm{~Gy}$ compared to patients who receive a dose between 20 to $30 \mathrm{~Gy}(\mathrm{OR}=1.5 ; 95 \% \mathrm{CI}=0.674$ to 3.339, $\mathrm{p}=0.012$ ) Table 3.

\section{Secondary outcomes}

Among patients who were treated with EFRT, 2 out of twenty nine (6.9\%) developed breast cancer and 1 patient

Table 4. Type of Radiation Exposure and Secondary Malignant Neoplasms (SMNs)

\begin{tabular}{|c|c|c|c|c|}
\hline \multirow[t]{2}{*}{$\begin{array}{l}\text { Type of Radiotherapy } \\
(\mathrm{N}=57)\end{array}$} & \multicolumn{3}{|c|}{$\begin{array}{c}\text { Secondary Malignant Neoplasm } \\
\text { No. }(\%)\end{array}$} & \multirow[t]{2}{*}{$\begin{array}{c}\text { Total } \\
\text { No. }(\%)\end{array}$} \\
\hline & Breast & Nasopharynx & Peritoneal Mesothelioma & \\
\hline EFRT (N=29) & $2(6.9 \%)$ & - & $1(3.4 \%)$ & $3(10.3 \%)$ \\
\hline IFRT $(\mathrm{N}=28)$ & $2(7.1 \%)$ & $1(3.6 \%)$ & - & $3(10.7 \%)$ \\
\hline Unknown $(\mathrm{N}=2)$ & - & - & - & - \\
\hline
\end{tabular}

EFRT, Extended field radiation therapy; IFRT, Involved field radiation therapy 
developed peritoneal mesothelioma (3.4\%). Following IFRT, 2 out of twenty eight $(7.1 \%)$ had breast cancer while 1 patient developed nasopharynx cancer (3.6\%) as shown in Table 4 . We observed no statistically significant difference between patients who received EFRT versus IFRT in the risk of developing SMNs (EFRT-to-IFRT ratio $[\mathrm{E} / \mathrm{I}]=1.040 ; 95 \% \mathrm{CI}=0.192$ to $5.647, \mathrm{p}=0.964)$.

\section{Discussion}

This population-based cohort study assessed one hundred and twelve patients with HL for the development of secondary cancers post-treatment from three teaching medical centers across Lebanon. Consistent with published literature in Lebanon on HL, our results showed that HL gender distribution is equal between both genders with a mean age at diagnosis of 30 years (Sader-Ghorraet al., 2014). Nodular sclerosis was the most common subtype of $\mathrm{HL}$ in our patient population $(56.3 \%)$ and in congruence with a study by Otrok et al. whereby nodular sclerosis constituted the major histological subtype of HL (64.1\%) in the assessed Lebanese patients (Otrock et al., 2013). This finding is also consistent with international data from other Arab countries and the United States (Otrock et al., 2013; NCCN, 2015). In the present study, the cumulative incidence value was $9.8 \%$. Despite our shorter follow-up duration (13 years), this value is in congruence with those reported in the literature from various European and North American institutions and cancer registries whereby the cumulative incidence values ranged from $6.5 \%$ to $17 \%$ at 20 years of follow-up and from $18 \%$ to $29.4 \%$ at 30 years (Dörffel et al., 2015). In a collaborative British cohort study, Swerdlow et al. described the 20-year cumulative risk of SMNs to be $13 \%$ for CHT only and $18 \%$ for combined modality (Swerdlow et al., 2011).

The incidence of SMNs after HL treatment has been assessed in various studies while taking into consideration the age at diagnosis, gender, treatment, and type of secondary cancers development. Constine et al. reported an increased risk of SMNs with female gender, increasing radiation dose, and age at treatment (Constine et al., 2008). This higher incidence of SMNs in women compared to men was also seen in our study. Similarly, and consistent with our findings, breast cancer was listed as the most common SMN with a lifetime cumulative risk between 25\% and 33\% (Lorigan et al., 2005; Constine et al., 2008; Dörffel et al., 2015). In an observational study with 33year follow-up, Schellong et al. showed that secondary breast cancer in HL women survivors had a cumulative incidence of $19 \%$ (95\%; CI $12 \%$ to $29 \%$ ) 30 years after treatment; that it is associated with RT doses between 20 to $45 \mathrm{~Gy}$ and age at which RT was administered (pubertal phase) (Schellong et al. 2014).

The interval to SMNs development is related to its histopathologic type. In our study, no secondary solid tumors occurred before 12 years. Constine et al. reported no solid tumors occurring before 8 years (Constine et al., 2008). Travis et al. showed an increase risk of lung cancer after 1 to 4 years of initial treatment with alkylating agents and 5 to 9 years after radiotherapy (Travis et al., 2002). Similarly, Swerdlow et al. showed that after CHT alone, the risks of lung cancer, NHL and leukemia peaked 5 to 9 years after therapy then diminished considerably while the risks of these and ten other types of cancer remained elevated for 25 years and longer after combined modality (Swerdlow et al., 2011).

Comparing the number of patients who developed SMNs between the two cohorts in our study, we found a similar number after combined modality and $\mathrm{CHT}$ alone ( $10 \%$ vs $9.4 \%$ ). In contrary, Swerdlow et al. reported that the relative risk of secondary cancers in HL survivors was much lower with CHT alone (RR, 2.0; 95\% CI, 1.7 to 2.4) compared to combined modalities (RR 3.9; $95 \%$ CI, 3.5 to 4.4) (Swerdlow et al., 2011). This discordance could be explained by the small sample size of our study and the inability to assess the impact of the selected chemotherapeutic agents along with exposure to radiation therapy in the development of SMNs.

The results of the present study showed that patients who received a combined therapy with a RT dose higher than 41 Gy had an OR of 1.5 times those who received combined therapy with a dose range between 20 and 30 Gy $(p=0.012)$. Contrary to our results, van Leeuwen et al. specified that breast cancer risk increased with increasing radiation dose among patients who received $\mathrm{RT}$ only $(\mathrm{RR}=$ $12.7,95 \% \mathrm{CI}=1.8$ to 86 , for patients receiving greater than $38.5 \mathrm{~Gy}$ ) but not among patients treated with combined modality (van Leeuwen et al., 2003). In line with our results, Koh et al. showed a reduction in excess relative risk (ERR) by $40 \%$ when the RT dose was dropped from 35 Gy to $20 \mathrm{~Gy}$ (Koh et al., 2007). Similarly, Constine et al. reported that SMNs risk increased with increasing doses of mantle radiation. This increase in risk was 2.5 fold for female patients treated with more than $35 \mathrm{~Gy}$ (Constine et al., 2008). Our findings confirm previous studies correlating the RT dose with SMNs development and negate those that raised doubt about this theory (Constine et al., 2008; O'Brien et al., 2010; Omer et al., 2012; Oeffinger et al., 2013; Dörffel et al., 2015).

Although we were expecting a decrease in radiation induced secondary malignancies with the transition to IFRT, our results were not statistically significant. Our results were consistent with a meta-analysis of 10 trials which showed no significant difference in secondary cancer development when comparing IFRT to EFRT (IFRT-to-EFRT 0R=1.17; p=0.28) (Franklin et al., 2006). Conversely, Hodgson et al. using a radiobiological model showed that the median predicted 20-year ERR of breast cancer for women treated at age 20 with 35 Gy mantle EFRT was 4.8 and it was predicted to decline to 1.8 after 35 Gy IFRT (Hodgson et al., 2007). In addition, Koh et al. showed a reduction in incidence for female breast and lung cancer by approximately $65 \%$ and for male lung cancer by approximately $35 \%$ while moving from 35 Gy mantle RT to 35 Gy IFRT (Koh et al., 2007). Similarly Sasse et al. confirmed that IFRT can replace EFRT while sustaining the long-term outcomes of HL patients, reducing the acute toxicities and possibly decreasing the risk of secondary malignancies (Sasse et al., 2012).

Our study is limited by some incomplete data as we may have lost patients that did indeed develop SMNs to relocation or follow-up treatment in other institutions, 
which would lead to underreporting of secondary cancers. Moreover, we could not estimate the impact of RT dose to the specific location where the cancer had developed and neither were we able to evaluate the effect of the RT dose and type (involved versus extended) simultaneously on secondary solid tumors. However, despite those limitations, we were able to show a dose response relationship between RT and SMNs. Finally, our findings on the risk of SMNs among HL survivors should be considered preliminary due to the relatively short duration of follow-up. Accordingly, this study sets the stage for a comprehensive, inter-institutional investigation that we plan to conduct which would include a larger population from multiple medical centers across the country and region.

Every year, between 600 to 700 new cases of HL and NHL are diagnosed in Lebanon (Sader-Ghorra et al., 2014). According to the Lebanese Cancer Registry, HL represents $22 \%$ of all diagnosed lymphomas (National Cancer Registry, 2004). Since the treatment protocols of HL are complex and continue to involve EFRT and IFRT in the Lebanese practice, our findings offer clinical implications for health care practitioners to follow newer evidence based guidelines for the use of RT in HL patients, develop national screening programs for HL survivors and provide this patient population with counseling services for the early detection of long-term complications of therapy particularly SMNs.

In conclusion, the results of our study confirmed that the risk of secondary cancer is higher among patients treated with combined therapy with a radiation dose greater than 31 Gy independently of the RT type used. Ultimately, large observational confirmatory studies will be needed to optimize RT dose of HL with regard to the development of secondary solid tumors while preserving outstanding cure outcomes.

\section{References}

Aleman BM, van den Belt-Dusebout AW, Klokman WJ, et al (2003). Long-term cause-specific mortality of patients treated for Hodgkin's disease. J Clin Oncol, 21, 3431-9.

Aleman B, Schaapveld M, van Eggermond AM, et al (2012). Second cancer risk 40 years after hodgkin lymphoma treatment. Int J Radiat Oncol Biol Phys, 84, S70-1.

Choi DK, Helenowski I, Hijya N (2014). Secondary malignancies in pediatric cancer survivors: Perspectives and review of the literature. Int J Cancer, 135, 1764-73.

Constine LS, Tarbell N, Hudson MM, et al (2008). Subsequent malignancies in children treated for Hodgkin's disease: associations with gender and radiation dose. Int $J$ Radiat Oncol Biol Phys, 72, 24-33.

Dörffel W, Riepenhausen M, Lüders H, Brämswig J, Schellong G (2015). Secondary malignancies following treatment for Hodgkin's Lymphoma in childhood and adolescence. Dtsch Arztebl Int, 112, 320-7.

Franklin J, Pluetschow A, Paus M, et al (2006). Second malignancy risk associated with treatment of Hodgkin's lymphoma: meta-analysis of the randomised trials. Ann Oncol, 17, 1749-60.

Gilbert ES, Stovall M, Gospodarowicz M, et al (2003). Lung cancer after treatment for Hodgkin's disease: focus on radiation effects. Radiat Res, 159, 161-73.
Hodgson DC, Koh ES, Tran TH, et al (2007) Individualized estimates of second cancer risks after contemporary radiation therapy for Hodgkin lymphoma. Cancer, 110, 2576-86.

Koh ES, Tran TH, Heydarian M, et al (2007). A comparison of mantle versus involved-field radiotherapy for Hodgkin's lymphoma: reduction in normal tissue dose and second cancer risk. Radiat Oncol, 2, 13.

Lorigan P, Radford J, Howell A, Thatcher N (2005). Lung cancer after treatment for Hodgkin's lymphoma: a systematic review. Lancet Oncol, 6, 773-79.

Matasar MJ and Zelenetz AD (2008). Overview of lymphoma diagnosis and management. Radiol Clin North Am, 46, 175-98.

National Comprehensive Cancer Network (2015). Hodgkin Lymphoma. Clinical Practice Guidelines in Oncology. National Comprehensive Cancer Network (V 2.2015). http://www.nccn.org/professionals/physician_gls/pdf/ hodgkins.pdf

National Cancer Registry (2004). Beirut: Ministry of Public Health. [Accessed 02/02/2014].

$\mathrm{Ng}$ AK, Bernardo MV, Weller E, et al (2002). Second malignancy after Hodgkin disease treated with radiation therapy with or without chemotherapy: long-term risks and risk factors. Blood, 100, 1989-96.

Noordijk EM, Carde P, Dupouy N, et al (2006). Combined modality therapy for clinical stage I or II Hodgkin's lymphoma: long-term results of the European Organisation for Research and Treatment of Cancer $\mathrm{H} 7$ randomized controlled trials. J Clin Oncol, 24, 3128-35.

O'Brien MM, Donaldson SS, Balise RR, Whittemore AS, and Link MP (2010). Second malignant neoplasms in survivors of pediatric Hodgkin's lymphoma treated with low-dose radiation and chemotherapy. J Clin Oncol, 28, 1232-9.

Oeffinger KC, Baxi SS, Friedman DN, Moskowitz CS (2013). Solid tumor second primary neoplasms: Who is at risk, what can we do? Semin Oncol, 40, 676-89.

Omer B, Kadan-Lottick NS, Roberts KB, et al (2012). Patterns of subsequent malignancies after Hodgkin lymphoma in children and adults. Br J Haematol, 5, 615-25.

Otrock ZK, Saab J, Aftimos G, et al (2013). A Collaborative nationwide Lymphoma study in Lebanon: Incidence of various subtypes and analysis of associations with viruses. Pathol Oncol Res, 19, 715-22.

Sader-Ghorra C, Rassy M, Naderi S, Kourie HR, Kattan J (2014). Type distribution of lymphomas in Lebanon: Five-year single institution experience. Asian Pac J Cancer Prev, 15, 5825-8.

Sasse S, Klimm B, Görgen H, et al (2012). Comparing longterm toxicity and efficacy of combined modality treatment including extended- or involved-field radiotherapy in earlystage Hodgkin's lymphoma. Ann Oncol, 23, 2953-9.

Schellong G, Riepenhausen M, Creutzig U, et al (1997). Low risk of secondary leukemias after chemotherapy without mechlorethamine in childhood Hodgkin's disease. J Clin Oncol, 25, 2241-53.

Schellong G, and Riepenhausen M, (2004). Late effects after therapy of Hodgkin's disease: Update 2003/04 on overwhelming postsplenectomy infections and secondary malignancies. Klin Pädiatr, 216, 364-9.

Schellong G, Riepenhausen M, Ehlert K, et al (2014). Breast cancer in young women after treatment for Hodgkin's disease during childhood or adolescence. Dtsch Arztebl Int, 111, 3-9.

Swerdlow AJ, Higgins CD, Smith P, et al (2011). Second cancer risk after chemotherapy for Hodgkin's Lymphoma: a collaborative British cohort study. J Clin Oncol, 29, 4096-104.

Travis LB, Gospodarowicz M, Curtis RE, et al (2002). Lung cancer following chemotherapy and radiotherapy for 


\section{Tamara Abou-Antoun et al}

Hodgkin's disease. J Natl Cancer Inst, 94, 182-92.

Travis LB, Hill DA, Dores GM, et al (2003). Breast cancer following radiotherapy and chemotherapy among young women with Hodgkin disease. JAMA, 290, 465-75.

van Leeuwen FE, Klokman WJ, Veer MB, et al (2000). Long term risk of second malignancy in survivors of Hodgkin's disease treated during adolescence of young adulthood. $J$ Clin Oncol, 18, 487-97.

van Leeuwen FE, Klokman WJ, Stovall M, et al (2003). Roles of radiation dose, chemotherapy, and hormonal factors in breast cancer following Hodgkin's disease. J Natl Cancer Inst, 95, 971-80.

Yahalom J (2009). Role of radiation therapy in Hodgkin's lymphoma. Cancer J, 15, 155-60. 